\title{
Bargmann invariants and geometric phases: A generalized connection
}

\author{
Eqab M. Rabei* \\ Department of Physics, Mutah University, PostBox 7, Karak, Jordan \\ Arvind $^{\dagger}$ \\ Department of Physics, Guru Nanak Dev University, Amritsar 143005, India \\ N. Mukunda \\ Centre for Theoretical Studies and Department of Physics, Indian Institute of Science, Bangalore 560012, India \\ R. Simon ${ }^{\S}$ \\ The Institute of Mathematical Sciences, C.I.T. Campus, Chennai 600113, India
}

\begin{abstract}
We develop the broadest possible generalization of the well known connection between quantummechanical Bargmann invariants and geometric phases. The key concept is that of null phase curves in quantum-mechanical ray and Hilbert spaces. Examples of such curves are developed. Our generalization is shown to be essential for properly understanding geometric phase results in the cases of coherent states and of Gaussian states. Differential geometric aspects of null phase curves are also briefly explored.
\end{abstract}

\section{INTRODUCTION}

The geometric phase was originally discovered in the context of cyclic adiabatic quantum-mechanical evolution, governed by the time-dependent Schrödinger equation with a Hermitian Hamiltonian operator [1,2]. Subsequent work has shown that many of these restrictions can be lifted. Thus the geometric phase can be defined in nonadiabatic [3], noncyclic and even nonunitary evolution [4]. Generalization to the non-Abelian case has also been achieved [5]. Finally the kinematic approach [6] demonstrated that even the Schrödinger equation and a Hamiltonian operator are not needed for defining the geometric phase. The intimate relationship between geometric phase and Hamilton's theory of turns [7] has also been brought out [8].

An important consequence of the kinematic approach has been to show clearly the close connection between geometric phases, and a family of quantum-mechanical invariants introduced by Bargmann [9] while giving a new proof of the Wigner [10] unitary-antiunitary theorem. This connection depends in an essential way upon the concept of free geodesics in quantum mechanical ray and Hilbert spaces, and the vanishing of geometric phases for these geodesics.

The purpose of this paper is to generalize this important link between Bargmann invariants and geometric phases to the broadest possible extent by going beyond the use of free geodesics. The key is to characterize in a complete way those ray space curves with the property that the geometric phase vanishes for any connected stretch of any one of them. We

\footnotetext{
*Electronic address: eqab@center.mutah.edu.jo

${ }^{\dagger}$ Electronic address: arvind@physics.iisc.ernet.in

*Electronic address: nmukunda@cts.iisc.ernet.in

${ }^{\S}$ Electronic address: simon@imsc.ernet.in
}

show that this property can be translated into an elementary and elegant statement concerning the inner product of any two Hilbert space vectors along any lift of such a ray space curve. We refer to these as "null phase curves," and the generalization of the familiar statement linking Bargmann invariants and geometric phases is achieved by replacing free geodesics by such curves. A free geodesic is always a null phase curve; however, the latter is much more general.

This paper is arranged as follows. Section II recalls the basic features of the kinematic approach to the geometric phase; sets up free geodesics in ray and Hilbert spaces; shows that the geometric phase for any free geodesic vanishes; introduces the Bargmann invariants; and describes their connection to geometric phases for ray space polygons bounded by free geodesics. In Sec. III it is argued that it should be possible to generalize this connection. This motivates the definition and complete characterization of null phase curves at the Hilbert space level, the previous free geodesics being a very specific case. It is then shown that such curves allow us to generalize the previously stated connection to the broadest possible extent. Section IV defines the concept of constrained geodesics in ray and Hilbert spaces, the motivation being that in some situations such curves may in fact be null phase curves. The idea is extremely simple, namely, we limit ourselves to some chosen submanifolds in ray (and Hilbert) space, and determine curves of minimum length lying within these submanifolds. Section V examines several interesting examples to illustrate these ideas: a submanifold arising out of a linear subspace of Hilbert space; coherent states for one degree of freedom; centered Gaussian pure states for one degree of freedom; and an interesting submanifold in the space of two-mode coherent states. It turns out that in the first case constrained geodesics are just free geodesics, while in the remaining cases they are very different. This shows that the generalized con- 
nection between Bargmann invariants and geometric phases presented in this paper is just what is needed to be physically interesting and appropriate. In Sec. VI we present a brief discussion of these ideas in the differential geometric framework natural to geometric phases, and also develop a direct ray space description of null phase curves. Section VII contains concluding remarks.

\section{CONNECTION BASED ON FREE GEODESICS}

Let $\mathcal{H}$ be the Hilbert space of states of some quantum system, $\mathcal{R}$ the associated ray space, and $\pi: \mathcal{H} \rightarrow \mathcal{R}$ the corresponding projection. We shall be dealing with (sufficiently) smooth parametrized curves $\mathcal{C}$ of unit vectors in $\mathcal{H}$, and their images $C$ in $\mathcal{R}$. A curve $\mathcal{C}$ is described as follows:

$$
\mathcal{C}=\left\{\psi(s) \in \mathcal{H} \mid\|\psi(s)\|=1 \quad s_{1} \leqslant s \leqslant s_{2}\right\} \subset \mathcal{H} .
$$

Its image $C$ is a curve of pure state density matrices:

$$
\begin{gathered}
\pi[\mathcal{C}]=C \subset \mathcal{R}, \\
C=\left\{\rho(s)=\psi(s) \psi(s)^{\dagger} \mid s_{1} \leqslant s \leqslant s_{2}\right\} .
\end{gathered}
$$

Any $\mathcal{C}$ in $\mathcal{H}$ projecting onto a given $C$ in $\mathcal{R}$ is a lift of the latter. In particular, we have a horizontal lift $\mathcal{C}^{(h)}$ if the vectors $\psi^{(h)}(s)$ along it are such that

$$
\left(\psi^{(h)}(s), \frac{d}{d s} \psi^{(h)}(s)\right)=0 .
$$

For any curve $C \subset \mathcal{R}$, a geometric phase $\varphi_{g}[C]$ is defined. Its calculation is facilitated by going to any lift $\mathcal{C}$, calculating the total and dynamical phases for $\mathcal{C}$, and taking the difference

$$
\begin{gathered}
\pi[\mathcal{C}]=C: \\
\varphi_{g}[C]=\varphi_{\text {tot }}[\mathcal{C}]-\varphi_{\text {dyn }}[\mathcal{C}], \\
\varphi_{\text {tot }}[\mathcal{C}]=\arg \left(\psi\left(s_{1}\right), \psi\left(s_{2}\right)\right), \\
\varphi_{\text {dyn }}[\mathcal{C}]=\operatorname{Im} \int_{s_{1}}^{s_{2}} d s\left(\psi(s), \frac{d}{d s} \psi(s)\right) .
\end{gathered}
$$

In particular, if $\mathcal{C}$ is horizontal $\varphi_{\text {dyn }}[\mathcal{C}]$ vanishes, and $\varphi_{g}[C]$ is just $\varphi_{\text {tot }}[\mathrm{C}]$.

Now we define free geodesics in $\mathcal{R}$ and $\mathcal{H}$. Given $C$ in $\mathcal{R}$ and any lift $\mathcal{C}$ in $\mathcal{H}$, the length of the former can be defined as the following nondegenerate functional:

$$
L[C]=\int_{s_{1}}^{s_{2}} d s\left\{\left\|\frac{d \psi(s)}{d s}\right\|^{2}-\|\left.\left(\psi(s), \frac{d \psi(s)}{d s}\right)\right|^{2}\right\}^{1 / 2} .
$$

It is easy to check that the integrand here is independent of the choice of lift $\mathcal{C}$; it leads to the well known Fubini-Study metric on $\mathcal{R}[11,12]$. Free geodesics in $\mathcal{R}$ are those $C$ 's for which $L[C]$ is a minimum for given end points. And by definition a free geodesic in $\mathcal{H}$ is any lift of a free geodesic in $\mathcal{R}$. It can be shown [6] that any free geodesic in $\mathcal{R}$ can be lifted to $\mathcal{H}$, and the parametrization chosen so that it can be described as follows:

$$
\begin{gathered}
\psi(s)=\phi_{1} \cos s+\phi_{2} \sin s \\
\left(\phi_{1}, \phi_{1}\right)=\left(\phi_{2}, \phi_{2}\right)=1, \quad\left(\phi_{1}, \phi_{2}\right)=0 .
\end{gathered}
$$

Thus we have here a plane two-dimensional curve determined by a pair of orthonormal vectors in $\mathcal{H}$, an arc of a circle. It may be helpful to make the following comment concerning free geodesics. Given any two "nonorthogonal" points $\rho_{1}, \rho_{2} \in \mathcal{R}$ such that $\operatorname{Tr}\left(\rho_{1} \rho_{2}\right) \neq 0$, we can always choose unit vectors $\psi_{1}, \psi_{2} \in \mathcal{H}$ projecting onto $\rho_{1}, \rho_{2}$ respectively, such that the inner product $\left(\psi_{1}, \psi_{2}\right)$ is real positive. Then the free geodesic (2.6) will connect $\psi_{1}$ and $\psi_{2}$ if we take $\phi_{1}=\psi_{1}$ and $\phi_{2}=\left[\psi_{2}-\psi_{1}\left(\psi_{1}, \psi_{2}\right)\right] /\left\{1-\left(\psi_{1}, \psi_{2}\right)^{2}\right\}^{1 / 2}$. It is now clear that $\psi(0)=\psi_{1}$, and $\psi(s)=\psi_{2}$ for $s$ $=\cos ^{-1}\left(\psi_{1}, \psi_{2}\right) \in(0, \pi / 2)$. It is clear that the curve in $\mathcal{H}$ given by Eq. (2.6) is horizontal and for any two points on it with $\left|s_{1}-s_{2}\right|<\pi / 2$, the inner product $\left(\psi\left(s_{1}\right), \psi\left(s_{2}\right)\right)$ is real positive, so $\psi\left(s_{1}\right)$ and $\psi\left(s_{2}\right)$ are in phase in the Pancharatnam sense [13]. From these properties of free geodesics, the result [6]

$$
\varphi_{g}[\text { free geodesic in } \mathcal{R}]=0
$$

follows. This can be exploited to connect geometric phases to Bargmann invariants.

Let $\psi_{1}, \psi_{2}, \ldots, \psi_{n}$ be any $n$ unit vectors in $\mathcal{H}$, no two consecutive ones being orthogonal, and let $\rho_{1}, \rho_{2}, \ldots, \rho_{n}$ be their images in $\mathcal{R}$. Then the corresponding $n$-vertex Bargmann invariant is defined as

$$
\begin{aligned}
\Delta_{n}\left(\psi_{1}, \psi_{2}, \ldots, \psi_{n}\right) & =\left(\psi_{1}, \psi_{2}\right)\left(\psi_{2}, \psi_{3}\right) \ldots\left(\psi_{n}, \psi_{1}\right) \\
& =\operatorname{Tr}\left(\rho_{1} \rho_{2} \ldots \rho_{n}\right) .
\end{aligned}
$$

Now we draw $n$ free geodesics in $\mathcal{R}$ connecting $\rho_{1}$ to $\rho_{2}, \rho_{2}$ to $\rho_{3}, \ldots, \rho_{n}$ to $\rho_{1}$. Thus we obtain an $n$-sided polygon in $\mathcal{R}$ bounded by free geodesics, and we can compute the corresponding geometric phase. Repeatedly exploiting Eq. (2.7) we obtain the basic result [6]:

$$
\begin{gathered}
\varphi_{g}\left[\begin{array}{c}
n-\text { vertex polygon in } \mathcal{R} \text { connecting } \rho_{1} \text { to } \rho_{2}, \\
\rho_{2} \text { to } \rho_{3}, \ldots, \rho_{n} \text { to } \rho_{1} \text { by free geodesics }
\end{array}\right] \\
=-\arg \Delta_{n}\left(\psi_{1}, \psi_{2}, \ldots, \psi_{n}\right) \\
\rho_{j}=\psi_{j} \psi_{j}^{\dagger}, j=1,2, \ldots, n
\end{gathered}
$$

We mention in passing that this result is of considerable conceptual as well as practical value [14].

In connection with the above result, the following remarks may be made. As is clear from Eq. (2.8), the phases of the individual vectors $\psi_{1}, \psi_{2}, \cdots, \psi_{n}$ can be freely altered. We need only assume that successive pairs of unit vectors are not mutually orthogonal; then the Bargmann invariant is nonzero and has a well defined phase.

\section{GENERALIZED CONNECTION}

The definition (2.8) of the Bargmann invariant requires only the choice of the $n$ vertices $\rho_{1}, \rho_{2}, \ldots, \rho_{n} \in \mathcal{R}$; consecutive ones need not be connected in any way to form a closed figure. This suggests that the connection (2.9) between these invariants and geometric phases may apply more generally, not only in the case where we connect $\rho_{1}$ to $\rho_{2}, \rho_{2}$ 
to $\rho_{3}, \ldots, \rho_{n}$ to $\rho_{1}$ by free geodesics. We now show that this is indeed so.

We need to characterize the most general (smooth) curves $C \subset \mathcal{R}$ having the property

$$
\varphi_{g}[\text { any connected portion of } C]=0 \text {. }
$$

We know that if $C$ is a free geodesic, this property does follow, but there may be (indeed there are) many other possibilities. We can develop a simple necessary and sufficient condition on $C$ such that Eq. (3.1) holds.

Given the curve $C \subset \mathcal{R}$, let $\mathcal{C}^{(h)}$ be a horizontal lift and $\mathcal{C}$ a general lift of $C$ in $\mathcal{H}$. We have

$$
\begin{aligned}
C & =\left\{\rho(s) \mid \rho(s)^{\dagger}=\rho(s) \geqslant 0, \quad \rho(s)^{2}=\rho(s), \quad \operatorname{Tr} \rho(s)=1, s_{1} \leqslant s \leqslant s_{2}\right\}, \\
\mathcal{C}^{(h)} & =\left\{\psi^{(h)}(s) \in \mathcal{H} \mid \pi\left(\psi^{(h)}(s)\right)=\rho(s), \quad\left(\psi^{(h)}(s), \frac{d}{d s} \psi^{(h)}(s)\right)=0\right\}, \\
\mathcal{C} & =\left\{\psi(s) \in \mathcal{H} \mid \psi(s)=e^{i \alpha(s)} \psi^{(h)}(s)\right\} .
\end{aligned}
$$

Here $\alpha(s)$ is some (smoothly varying) phase angle. For any two points on $C$ with parameter values $s$ and $s^{\prime}>s$ we have

$$
\begin{aligned}
\varphi_{g}\left[\rho(s) \text { to } \rho\left(s^{\prime}\right) \text { along } C\right] & =\varphi_{\text {tot }}\left[\psi^{(h)}(s) \text { to } \psi^{(h)}\left(s^{\prime}\right) \text { along } \mathcal{C}^{(h)}\right]=\arg \left(\psi^{(h)}(s), \psi^{(h)}\left(s^{\prime}\right)\right) \\
& =\arg \left(e^{-i \alpha(s)} \psi(s), e^{-i \alpha\left(s^{\prime}\right)} \psi\left(s^{\prime}\right)\right)=\arg \left(\psi(s), \psi\left(s^{\prime}\right)\right)+\alpha(s)-\alpha\left(s^{\prime}\right) .
\end{aligned}
$$

From this result we see that the necessary and sufficient condition on $C$ to secure the property (3.1) can be expressed in several equivalent ways, using either an arbitrary lift $\mathcal{C}$ of $C$ or a horizontal lift $\mathcal{C}^{(h)}$ :

$\varphi_{g}[$ any connected portion of $C]=0 \Leftrightarrow \arg \left(\psi(s), \psi\left(s^{\prime}\right)\right)=\alpha\left(s^{\prime}\right)-\alpha(s)$, any $s^{\prime}$ and $s$

$$
\begin{aligned}
& \Leftrightarrow \frac{\partial^{2}}{\partial s^{\prime} \partial s} \arg \left(\psi(s), \psi\left(s^{\prime}\right)\right)=0 \\
& \Leftrightarrow \arg \left(\psi(s), \psi\left(s^{\prime}\right)\right)=\text { separable in } s^{\prime} \text { and } s \\
& \Leftrightarrow\left(\psi^{(h)}(s), \psi^{(h)}\left(s^{\prime}\right)\right)=\text { real positive, any } s^{\prime} \text { and } s \\
& \Leftrightarrow \text { any two points of } \mathcal{C}^{(h)} \text { are in phase. }
\end{aligned}
$$

Here, separability is to be understood in the additive, and not in the multiplicative, sense. It is important to recognize that these characterizations are reparametrization invariant. Any curve $C \subset \mathcal{R}$ obeying (3.4) will be called a "null phase curve in $\mathcal{R}$,' and any lift $\mathcal{C}$ of such a $C$ will be called a "null phase curve in $\mathcal{H}$.' 'Free geodesics are null phase curves, but the opposite is not necessarily true.

It may be helpful to make some additional remarks at this point to clarify the ideas involved. If a curve $\mathcal{C} \subset \mathcal{H}$ is such that any two points on it (not too far apart) are in phase, then it is definitely horizontal:

$\mathcal{C}=\{\psi(s)\}:\left(\psi(s), \psi\left(s^{\prime}\right)\right)=$ real positive

$$
\begin{aligned}
& \Rightarrow\left(\psi(s), \frac{d \psi\left(s^{\prime}\right)}{d s^{\prime}}\right)=\text { real } \\
& \Rightarrow\left(\psi(s), \frac{d \psi(s)}{d s}\right)=0 \\
& \Rightarrow \mathcal{C} \text { horizontal. }
\end{aligned}
$$

The image $C=\pi[\mathcal{C}]$ is obviously a null phase curve in $\mathcal{R}$, since Eq. (3.4) is obeyed with $\alpha(s)=0$; therefore, $\mathcal{C}$ being a lift of $C$ is also a null phase curve in $\mathcal{H}$. On the other hand, for a horizontal curve $\mathcal{C}^{(h)} \subset \mathcal{H}$, only "nearby points", are in phase:

$$
\begin{aligned}
\mathcal{C}^{(h)}= & \left\{\psi^{(h)}(s)\right\}=\text { horizontal } \\
& \Rightarrow\left(\psi^{(h)}(s), \frac{d}{d s} \psi^{(h)}(s)\right)=0, \\
& \Rightarrow\left(\psi^{(h)}(s), \psi^{(h)}(s+\delta s)\right) \simeq 1+0(\delta s)^{2}, \\
& \Rightarrow \arg \left(\psi^{(h)}(s), \psi^{(h)}(s+\delta s)\right)=0(\delta s)^{2} .
\end{aligned}
$$

However, two general points on $\mathcal{C}^{(h)}$ may well not be in phase, as $\arg \left(\psi(s), \psi\left(s^{\prime}\right)\right)$ could be nonzero. Hence $\mathcal{C}^{(h)}$ and its image $\pi\left[\mathcal{C}^{(h)}\right]$ may not be null phase curves. For $\pi\left[\mathcal{C}^{(h)}\right]$ to be a null phase curve, in addition to being horizontal (a 
local property), $\mathcal{C}^{(h)}$ must possess the global property that for general $s$ and $s^{\prime}$ the inner product $\left(\psi^{(h)}(s), \psi^{(h)}\left(s^{\prime}\right)\right)$ is real positive. This is what is captured in conditions (3.4).

We can now generalize the result (2.9) and strengthen it as follows. Given $n$ unit vectors $\psi_{1}, \psi_{2}, \ldots, \psi_{n} \in \mathcal{H}$ with images $\rho_{1}, \rho_{2}, \ldots, \rho_{n} \in \mathcal{R}$, draw any null phase curves joining consecutive pairs of points $\rho_{1}$ to $\rho_{2}, \rho_{2}$ to $\rho_{3}, \ldots, \rho_{n}$ to $\rho_{1}$. (This can certainly be done since in any event free geodesics are available.) Then, by exactly the same arguments that lead to the connection (2.9) we obtain $\varphi_{g}\left[\begin{array}{c}n-\text { sided figure in } \mathcal{R} \text { with vertices } \rho_{1}, \rho_{2}, \ldots, \rho_{n} \\ \text { and bounded by null phase curves }\end{array}\right]=-\arg \Delta_{n}\left(\psi_{1}, \psi_{2}, \ldots, \psi_{n}\right)$
It must be clear that this is the broadest generalization of the connection (2.9) that one can obtain. We see that we can replace each free geodesic belonging to a polygon in $\mathcal{R}$ by any null phase curve, and the geometric phase remains the same, since the right-hand side of Eq. (3.7) depends on the vertices alone.

\section{CONSTRAINED GEODESICS AS NULL PHASE CURVES}

We have seen that every free geodesic is a null phase curve, but the converse is generally not true. Nevertheless, the former fact inspires the following question: Can we alter the definition of a free geodesic, based on minimizing the length functional $L[C]$ of Eq. (2.5), in a natural way to obtain other kinds of geodesics, and will they turn out to be null phase curves as well?

The generalization we explore is the following: instead of dealing with curves (of unit vectors) in the complete Hilbert and ray spaces $\mathcal{H}$ and $\mathcal{R}$, we restrict ourselves to some (smooth) submanifold $M \subset \mathcal{R}$ and consider only curves $C$ lying in $M$ and connecting pairs of points in $M$. For such curves we minimize $L[C]$ with respect to variations of $C$ which stay within $M$. The resulting curves will naturally be called "constrained geodesics," and the question is, do constrained geodesics in some cases turn out to be null phase curves?

We emphasize that our question is not whether every null phase curve is a constrained geodesic lying in a suitably chosen submanifold $M \subset \mathcal{R}$ but, rather, whether the latter curves sometimes have the former property. The physically important examples presented in the next section show that our question is indeed interesting. In this section we set up the general framework to handle constrained geodesics in ray space.

Given $\mathcal{H}$ and $\mathcal{R}$ with $\operatorname{dim} \mathcal{H}=\operatorname{dim} \mathcal{R}+1$ in the real sense, we consider a submanifold $M \subset \mathcal{R}$ of $n$ (real) dimensions consisting of a (sufficiently smooth) family of unit rays, with (local) real independent and essential coordinates $\xi$ $=\left(\xi^{\mu}\right), \mu=1,2, \ldots, n$ :

$$
M=\left\{\rho(\xi) \in \mathcal{R} \mid \xi \in \mathfrak{R}^{n}\right\} \subset \mathcal{R} .
$$

(We do not indicate explicitly the domain in $\mathfrak{R}^{n}$ over which $\xi$ may vary.) The inverse image of $M$ in $\mathcal{H}$ will bring in an extra phase angle $\alpha$, and is denoted by $\mathcal{M}$ :

$$
\begin{aligned}
\mathcal{M} & =\pi^{-1}[M]=\{\psi(\xi ; \alpha) \in \mathcal{H} \mid \pi[\psi(\xi ; \alpha)]=\rho(\xi), \psi(\xi ; \alpha) \\
& \left.=e^{i \alpha} \psi(\xi ; 0)\right\} .
\end{aligned}
$$

(Of course each $\psi(\xi ; \alpha)$ is a unit vector, and $\alpha$ and $\xi^{\mu}$ taken together are local coordinates for $\mathcal{M}$.) So in the real sense $\operatorname{dim} \mathcal{M}=n+1$, and to avoid trivialities we must have 1 $+n / 2<$ complex dimension of $\mathcal{H}$.

Now we consider a parametrized curve $C \subset M \subset \mathcal{R}$, obtained by making the $n$ real variables $\xi^{\mu}$ into functions of a real parameter $s$ :

$$
C=\left\{\rho(\xi(s)), s_{1} \leqslant s \leqslant s_{2}\right\} \subset M .
$$

To lift $C$ to some $\mathcal{C} \subset \mathcal{M} \subset \mathcal{H}$, some (smooth) choice of phase angle $\alpha(s)$ as a function of $s$ must be made, and then we have

$$
\begin{gathered}
\mathcal{C}=\{\Psi(s)=\psi(\xi(s) ; \alpha(s))\} \subset \mathcal{M}, \\
\pi[\mathcal{C}]=C .
\end{gathered}
$$

Using the definition (2.5) the length $L[C]$ can be seen to involve only the partial derivatives of $\psi(\xi ; \alpha)$ with respect to the $\xi^{\mu}$, the dependence on $\alpha$ being trivial and not contributing at all. Therefore, we define

$$
\begin{gathered}
u_{\mu}(\xi ; \alpha)=\frac{\partial}{\partial \xi^{\mu}} \psi(\xi ; \alpha), \quad \mu=1,2, \ldots, n ; \\
u_{\mu}^{\perp}(\xi ; \alpha)=u_{\mu}(\xi ; \alpha)-\psi(\xi ; \alpha)\left(\psi(\xi ; \alpha), u_{\mu}(\xi ; \alpha)\right) .
\end{gathered}
$$

Normalization of $\psi(\xi ; \alpha)$ to unity for all $\xi$ and $\alpha$ implies

$$
\operatorname{Re}\left(\psi(\xi ; \alpha), u_{\mu}(\xi ; \alpha)\right)=0
$$

Now $L[C]$ can be expressed as follows:

$$
\begin{gathered}
L[C]=\int_{s_{1}}^{s_{2}} d s \sqrt{\|\dot{\Psi}(s)\|^{2}-|(\Psi(s), \dot{\Psi}(s))|^{2}} \\
=\int_{s_{1}}^{s_{2}} d s \sqrt{g_{\mu \nu}(\xi) \xi^{\mu} \xi^{\nu}}, \\
g_{\mu \nu}(\xi)=\operatorname{Re}\left(u_{\mu}^{\perp}(\xi ; \alpha), u_{\nu}^{\perp}(\xi ; \alpha)\right), \\
\xi^{\mu}=\xi^{\mu}(s) .
\end{gathered}
$$


The parameter dependences of $\xi$ and $\alpha$ are as in Eq. (4.4). From the essentiality of $\xi^{\mu}$ as coordinates for $M$, and the positivity of the metric on $\mathcal{H}$, one easily obtains the following results: the $n \times n$ matrix $\left\{\left(u_{\mu}^{\perp}(\xi ; \alpha), u_{\nu}^{\perp}(\xi ; \alpha)\right)\right\}$ is Hermitian positive definite and independent of $\alpha$; and only its real part $\left[g_{\mu \nu}(\xi)\right]$, which is symmetric positive definite, enters into $L[C]$.

To obtain the differential equations for constrained geodesics, we minimize $L[C]$ with respect to variations in $C$ that stay within $M$. This amounts to minimizing $L[C]$ in the final form given in Eq. (4.7), by making independent variations in the $n$ real functions $\xi^{\mu}(s)$; the result is well known from Riemannian geometry. After making a suitable choice of the parameter $s$ (affine parametrization), the differential equations for constrained geodesics become

$$
\begin{gathered}
\ddot{\xi}^{\mu}(s)+\Gamma_{\nu \lambda}^{\mu}[\xi(s)] \dot{\xi}^{\nu}(s) \dot{\xi}^{\lambda}(s)=0, \\
\Gamma_{\nu \lambda}^{\mu}(\xi)=\frac{1}{2} g^{\mu \rho}(\xi)\left[g_{\rho \nu, \lambda}(\xi)+g_{\rho \lambda, \nu}(\xi)-g_{\nu \lambda, \rho}(\xi)\right], \\
{\left[g^{\mu \nu}(\xi)\right]=\left[g_{\mu \nu}(\xi)\right]^{-1},} \\
g_{\rho \nu, \lambda}(\xi)=\frac{\partial g_{\rho \nu}(\xi)}{\partial \xi^{\lambda}}
\end{gathered}
$$

Here the $\Gamma$ 's are the familiar symmetric Christoffel symbols determined by the "metric" tensor $g_{\mu \nu}(\xi)$. Change in scale and shift of origin are the only remaining freedoms in choices for parameter $s$. It is a consequence of the differential equations above that

$$
g_{\mu \nu}[\xi(s)] \dot{\xi}^{\mu}(s) \dot{\xi}^{\nu}(s)=\text { const. }
$$

A general solution to Eq. (4.8) is uniquely determined by choices of initial values $\xi^{\mu}(0), \dot{\xi}^{\mu}(0)$. The resulting $\xi^{\mu}(s)$ determine some constrained geodesic $C \subset M \subset \mathcal{R}$, and for any (smooth) choice of $\alpha(s)$ we get a lift $\mathcal{C} \subset \mathcal{M} \subset \mathcal{H}$, which by definition is a constrained geodesic in $\mathcal{H}$. The meaning of the "conservation law" (4.9) in terms of Hilbert space vectors is interesting. In terms of the derivative of $\Psi(s)$ with respect to $s$, and its component orthogonal to $\Psi(s)$,

$$
\begin{aligned}
\dot{\Psi}(s)= & \frac{d}{d s} \psi(\xi(s) ; \alpha(s))=\dot{\xi}^{\mu}(s) u_{\mu}[\xi(s) ; \alpha(s)] \\
& +i \dot{\alpha}(s) \Psi(s),
\end{aligned}
$$

$$
\dot{\Psi}^{\perp}(s)=\dot{\Psi}(s)-\Psi(s)(\Psi(s), \dot{\Psi}(s))=\dot{\xi}^{\mu}(s) u_{\mu}^{\perp}[\xi(s) ; \alpha(s)],
$$

we have

$$
g_{\mu \nu}[\xi(s)] \dot{\xi}^{\mu}(s) \dot{\xi}^{\nu}(s)=\mathrm{const} \Rightarrow\left\|\dot{\Psi}^{\perp}(s)\right\|=\text { const. }
$$

We can then, if we wish, adjust the scale of $s$ so that $\dot{\Psi}^{\perp}$ becomes a unit vector for all $s$.

Having set up the basic formalism to determine constrained geodesics, in the next section we look at some physically motivated examples to see whether they are sometimes null phase curves as well.

\section{APPLICATIONS}

We look at four examples to illustrate the use of constrained geodesics in the geometric phase context, and to show the distinction in general between them and null phase curves.

\section{A. Subspaces of $\mathcal{H}$}

Let $\mathcal{H}_{0}$ be a linear subspace of $\mathcal{H}$ (as a complex vector space), and denote by $\mathcal{M} \subset \mathcal{H}_{0}$ the subset of unit vectors in $\mathcal{H}_{0}$. By projection, we obtain the submanifold $M$ $=\pi[\mathcal{M}] \subset \mathcal{R}$, with the real dimension of $M$ equal to $2 \times\left\{\left(\right.\right.$ complex dimension of $\left.\left.\mathcal{H}_{0}\right)-1\right\}$. In this case, constrained geodesics in $M$ happen to be free geodesics. Given any two (nonorthogonal) in-phase unit vectors in $\mathcal{M}$, say $\psi_{1}$ and $\psi_{2}$, the free geodesic connecting them, namely, from Eq. (2.6) the curve $\mathcal{C}$ consisting of the vectors

$$
\psi(s)=\psi_{1} \cos s+\frac{\left(\psi_{2}-\left(\psi_{1}, \psi_{2}\right) \psi_{1}\right)}{\sqrt{1-\left(\psi_{1}, \psi_{2}\right)^{2}}} \sin s,
$$

passes entirely through points of $\mathcal{M}$. Hence its image $\pi[\mathcal{C}]$ $=C$ lies entirely within $M$ and, being the free geodesic connecting $\pi\left(\psi_{1}\right)$ to $\pi\left(\psi_{2}\right)$, it must be the constrained geodesic as well. In this case, therefore, we do not get anything new.

Conversely, we see that to have a situation where constrained geodesics are different from free ones, the submanifold $M \subset \mathcal{R}$ must not arise from a subspace of $\mathcal{H}$ in the above manner. We now look at two such cases, of obvious physical importance, in which true generalizations of the original Bargmann invariant-geometric phase connection appear.

\section{B. Single mode coherent states}

We consider the family of coherent states for a single degree of freedom, described by Hermitian operators $\hat{q}, \hat{p}$ or the non-Hermitian combinations $\hat{a}, \hat{a}^{\dagger}$ :

$$
\begin{gathered}
\hat{a}=\frac{1}{\sqrt{2}}(\hat{q}+i \hat{p}), \quad \hat{a}^{\dagger}=\frac{1}{\sqrt{2}}(\hat{q}-i \hat{p}), \\
{[\hat{q}, \hat{p}]=i, \quad\left[\hat{a}, \hat{a}^{\dagger}\right]=1 .}
\end{gathered}
$$

A general normalized coherent state is labeled with a complex number $z$ and is generated by applying a unitary phase space displacement operator to the (Fock) vacuum state $|0\rangle$ :

$$
|z\rangle=\exp \left(z \hat{a}^{\dagger}-z^{*} \hat{a}\right)|0\rangle=\exp \left(-\frac{1}{2} z^{*} z+z \hat{a}^{\dagger}\right)|0\rangle,
$$

$$
\hat{a}|z\rangle=z|z\rangle .
$$

To conform to the notations of the preceding section, we introduce real parameters $\xi_{1}, \xi_{2}$, include a phase angle $\alpha$, and express the above states in terms of $\hat{q}$ and $\hat{p}$ as follows (for ease in writing we use $\xi_{1,2}$ rather than $\xi^{1,2}$ ):

$$
z=\frac{1}{\sqrt{2}}\left(\xi_{1}+i \quad \xi_{2}\right), \xi_{1,2} \epsilon \Re:
$$




$$
\begin{aligned}
\psi(\xi ; \alpha) & =e^{i \alpha}|z\rangle=\exp \left[i \alpha+i\left(\xi_{2} \hat{q}-\xi_{1} \hat{p}\right)\right]|0\rangle \\
& =\exp \left(i \alpha-\frac{i}{2} \xi_{1} \xi_{2}\right) \exp \left(i \xi_{2} \hat{q}\right) \exp \left(-i \xi_{1} \hat{p}\right)|0\rangle \\
& =\exp \left(i \alpha+\frac{i}{2} \xi_{1} \xi_{2}\right) \exp \left(-i \xi_{1} \hat{p}\right) \exp \left(i \xi_{2} \hat{q}\right)|0\rangle
\end{aligned}
$$

(Note that, as in Eq. (4.2), $\psi(\xi ; \alpha)$ is a vector in $\mathcal{H}$ parametrized by $\xi$ and $\alpha$, not a wave function.) These various equivalent forms facilitate further calculations.

The expectation values of $\hat{q}$ and $\hat{p}$ in these states are

$$
\begin{gathered}
(\psi(\xi ; \alpha), \hat{q} \psi(\xi ; \alpha))=\xi_{1}, \\
(\psi(\xi ; \alpha), \hat{p} \psi(\xi ; \alpha))=\xi_{2} .
\end{gathered}
$$

Now we compute the vectors $u_{\mu}(\xi ; \alpha)$ and their projections $u_{\mu}^{\perp}(\xi ; \alpha)$ orthogonal to $\psi(\xi ; \alpha)$, as defined in Eq. (4.5):

$$
\begin{gathered}
u_{1}=\partial_{1} \psi=-i\left(\hat{p}-\frac{1}{2} \xi_{2}\right) \psi, \\
u_{2}=\partial_{2} \psi=i\left(\hat{q}-\frac{1}{2} \xi_{1}\right) \psi ; \\
u_{1}^{\perp}=-i\left(\hat{p}-\xi_{2}\right) \psi, \quad u_{2}^{\perp}=i\left(\hat{q}-\xi_{1}\right) \psi .
\end{gathered}
$$

Here we used Eq. (5.5), and, for simplicity, omitted the arguments $\xi, \alpha$ in $\psi, u_{\mu}, u_{\mu}^{\perp}$. The inner products among the $u_{\mu}^{\perp}$ involve the fluctuations in $\hat{q}$ and $\hat{p}$ and the cross term. After easy calculations we find

$$
\begin{aligned}
& \left(u_{1}^{\perp}, u_{1}^{\perp}\right)=\left(\psi,\left(\hat{p}-\xi_{2}\right)^{2} \psi\right)=(\Delta p)^{2}=1 / 2, \\
& \left(u_{1}^{\perp}, u_{2}^{\perp}\right)=-\left(\psi,\left(\hat{p}-\xi_{2}\right)\left(\hat{q}-\xi_{1}\right) \psi\right)=i / 2, \\
& \left(u_{2}^{\perp}, u_{2}^{\perp}\right)=\left(\psi,\left(\hat{q}-\xi_{1}\right)^{2} \psi\right)=(\Delta q)^{2}=1 / 2 .
\end{aligned}
$$

Therefore, the induced metric tensor in the $\xi_{1}-\xi_{2}$ plane, defined in Eq. (4.7), is

$$
g_{\mu \nu}(\xi)=\frac{1}{2} \delta_{\mu \nu},
$$

namely, it is the ordinary Euclidean metric on $\mathfrak{R}^{2}$. Constrained geodesics in this case are just determined by straight lines in the $\xi$ plane, since all $\Gamma$ 's vanish:

$$
\begin{gathered}
z(s)=z_{0}+z_{1} s, \quad z_{0,1}=\frac{1}{\sqrt{2}}\left(q_{0,1}+i \quad p_{0,1}\right): \\
\xi_{1}(s)=q_{0}+q_{1} s, \quad \xi_{2}(s)=p_{0}+p_{1} s .
\end{gathered}
$$

At the Hilbert space level, a constrained geodesic $\mathcal{C}_{\text {constr.geo. }}$ can be taken to be a curve within the family of coherent states

$$
\mathcal{C}_{\text {constr.geo. }}=\left\{\Psi(s)=\left|z_{0}+z_{1} s\right\rangle\right\} .
$$

[Here we have omitted an $s$-dependent phase $\alpha(s)$.] Each vector $\Psi(s)$ along this curve is a (pure) coherent state, and cannot be written as a linear combination of two fixed states as in Eq. (2.6); so it is immediately clear that this is not a free geodesic at all.

Now we examine whether this constrained geodesic is a null phase curve. We find, using the criterion (3.4):

$$
\begin{aligned}
\arg \left(\Psi(s), \Psi\left(s^{\prime}\right)\right) & =\arg \left\langle z_{0}+z_{1} s \mid z_{0}+z_{1} s^{\prime}\right\rangle \\
& =\arg \left[\exp \left\{\left(z_{0}^{*}+z_{1}^{*} s\right)\left(z_{0}+z_{1} s^{\prime}\right)\right\}\right] \\
& =\arg \left[\exp \left(z_{0}^{*} z_{1} s^{\prime}+z_{0} z_{1}^{*} s\right)\right] \\
& =\left(s^{\prime}-s\right) \operatorname{Im} z_{0}^{*} z_{1} .
\end{aligned}
$$

This is a separable function of $s^{\prime}$ and $s$, so we do have a null phase curve. We can go from the above $\mathcal{C}_{\text {constrgeo. }}$ to a horizontal curve by adding a phase:

$$
\mathcal{C}_{\text {constr.geo. }}^{(h)}=\left\{\Psi^{\prime}(s)=\exp \left(-i s \operatorname{Im} z_{0}^{*} z_{1}\right) \Psi(s)\right\},
$$

and then we find that any two points on this curve are in phase, as expected.

The generalized connection (3.7) in this example now states: if $\left|z_{1}\right\rangle,\left|z_{2}\right\rangle, \ldots,\left|z_{n}\right\rangle$ are any $n$ pure coherent states given by choosing $n$ points in the complex plane, and we join these points successively by straight lines in the complex plane so that all along in Hilbert space we deal with individual coherent states and never with superpositions of them, we have

$$
\begin{aligned}
& \varphi_{g}\left[\begin{array}{l}
n \text {-sided plane polygon with vertices } \\
\text { at the coherent states } z_{1}, z_{2}, \ldots, z_{n}
\end{array}\right] \\
& \quad=-\arg \Delta_{n}\left(\left|z_{1}\right\rangle,\left|z_{2}\right\rangle, \ldots,\left|z_{n}\right\rangle\right) .
\end{aligned}
$$

The case $n=3$ leads to the area formula for the geometric phase for a triangle in the plane, a very familiar result [15]. From our point of view, the present example is a significant generalization of the original connection (2.9).

Going further, it is easy to convince oneself that in this example the most general null phase curve arises in the above manner; in other words, a given one-parameter family of coherent states $\{|z(s)\rangle\}$ obeys the separability condition (3.4) if and only if $\operatorname{Im} z(s)$ is a linear inhomogeneous expression in $\operatorname{Re} z(s)$, so that $z(s)$ describes a straight line in the complex plane as $s$ varies.

\section{Centered Gaussian pure states}

This example again deals with one canonical pair $\hat{q}, \hat{p}$. It is now more convenient to work with wave functions in the Schrödinger representation, and not with abstract ket vectors. The submanifold $\mathcal{M \subset \mathcal { H }}$ consists of normalized Gaussian wave functions parametrized by two real variables $\xi_{1}, \xi_{2}$ and a phase angle $\alpha$ defined as follows:

$$
\begin{gathered}
\psi(\xi ; \alpha ; q)=\left(\frac{\xi_{2}}{\pi}\right)^{1 / 4} \exp \left\{i \alpha+\frac{i}{2}\left(\xi_{1}+i \quad \xi_{2}\right) q^{2}\right\}, \\
\xi_{1} \in(-\infty, \infty), \quad \xi_{2} \in(0, \infty), \quad \alpha \in[0,2 \pi) .
\end{gathered}
$$


Normalizability requires that $\xi_{2}$ be strictly positive, so the combination $\xi_{1}+i \xi_{2}$ is a variable point in the upper half complex plane. The wave functions $u_{\mu}(\xi ; \alpha ; q)$ are

$$
\begin{gathered}
u_{1}(\xi ; \alpha ; q)=\frac{\partial}{\partial \xi_{1}} \psi(\xi ; \alpha ; q)=\frac{i}{2} q^{2} \psi(\xi ; \alpha ; q), \\
u_{2}(\xi ; \alpha ; q)=\frac{\partial}{\partial \xi_{2}} \psi(\xi ; \alpha ; q)=\frac{1}{2}\left(-q^{2}+\frac{1}{2 \xi_{2}}\right) \psi(\xi ; \alpha ; q) .
\end{gathered}
$$

It is clear that to obtain the components $u_{\mu}^{\perp}$ of $u_{\mu}$ orthogonal to $\psi$, and later to compute the inner products $\left(u_{\mu}^{\perp}, u_{\nu}^{\perp}\right)$, we need the expectation values of $q^{2}$ and $q^{4}$ in the state $\psi$. These are (omitting for simplicity the arguments of $\psi$ ):

$$
\begin{aligned}
& \left(\psi, q^{2} \psi\right)=\left(\frac{\xi_{2}}{\pi}\right)^{1 / 2} \int_{-\infty}^{\infty} d q q^{2} e^{-\xi_{2} q^{2}}=\frac{1}{2 \xi_{2}}, \\
& \left(\psi, q^{4} \psi\right)=\left(\frac{\xi_{2}}{\pi}\right)^{1 / 2} \int_{-\infty}^{\infty} d q q^{4} e^{-\xi_{2} q^{2}}=\frac{3}{4 \xi_{2}^{2}} .
\end{aligned}
$$

Now the necessary inner products and projections are easily found:

$$
\begin{gathered}
\left(\psi, u_{1}\right)=\frac{i}{4 \xi_{2}}, \quad\left(\psi, u_{2}\right)=0 \\
u_{1}^{\perp}=\frac{i}{2}\left(q^{2}-\frac{1}{2 \xi_{2}}\right) \psi, u_{2}^{\perp}=u_{2}=-\frac{1}{2}\left(q^{2}-\frac{1}{2 \xi_{2}}\right) \psi \\
\left(u_{1}^{\perp}, u_{1}^{\perp}\right)=\frac{1}{4}\left[\psi,\left(q^{2}-\frac{1}{2 \xi_{2}}\right)^{2} \psi\right]=\frac{1}{8 \xi_{2}^{2}} ; \\
\left(u_{1}^{\perp}, u_{2}^{\perp}\right)=\frac{i}{4}\left[\psi,\left(q^{2}-\frac{1}{2 \frac{\xi_{2}}{2}}\right)^{2} \psi\right]=\frac{i}{8 \xi_{2}^{2}} \\
\left(u_{2}^{\perp}, u_{2}^{\perp}\right)=\left(u_{2}, u_{2}\right)=\frac{1}{4}\left[\psi,\left(q^{2}-\frac{1}{2 \xi_{2}}\right)^{2} \psi\right]=\frac{1}{8 \xi_{2}^{2}} .
\end{gathered}
$$

From these results we obtain the induced metric over $M$ $=\pi[\mathcal{M}] \subset R$, described in the upper half complex plane by the metric tensor

$$
g_{\mu \nu}(\xi)=\operatorname{Re}\left(u_{\mu}^{\perp}(\xi ; \alpha), u_{\nu}^{\perp}(\xi ; \alpha)\right)=\frac{1}{8 \xi_{2}^{2}} \delta_{\mu \nu} .
$$

This is the well known form of the Lobachevskian metric in this model of Lobachevsky space [16]. Dropping the numerical factor $\frac{1}{8}$ for simplicity, the line element in the upper half plane is given by

$$
d s^{2}=\frac{1}{\xi_{2}^{2}}\left(d \xi_{1}^{2}+d \xi_{2}^{2}\right)
$$

and we must find the corresponding geodesics.

First we compute the nonvanishing $\Gamma$ 's. The inverse of $\left[g_{\mu \nu}(\xi)\right]$ has components

$$
g^{11}(\xi)=g^{22}(\xi)=\xi_{2}^{2}, g^{12}(\xi)=0 .
$$

We easily find that the nonvanishing $\Gamma$ 's are

$$
\Gamma_{12}^{1}(\xi)=\Gamma_{22}^{2}(\xi)=-\Gamma_{11}^{2}(\xi)=-\frac{1}{\xi_{2}} .
$$

Using these in Eq. (4.8) we find the following ordinary differential equations to determine geodesics:

$$
\begin{gathered}
\ddot{\xi}_{1}-\frac{2}{\xi_{2}} \dot{\xi}_{1} \dot{\xi}_{2}=0, \\
\ddot{\xi}_{2}+\frac{1}{\xi_{2}}\left(\dot{\xi}_{1}^{2}-\dot{\xi}_{2}^{2}\right)=0 .
\end{gathered}
$$

We can exploit the fact that these differential equations lead to the consequence

$$
\frac{1}{\xi_{2}^{2}}\left(\xi_{1}^{2}+\xi_{2}^{2}\right)=\text { const }
$$

the value of the constant depending on the particular geodesic. After elementary analysis, we find that there are two families of geodesics:

$$
\begin{array}{cl}
\text { Type I: } \quad \xi_{1}=\text { const, } \quad \xi_{2}=a e^{b s}, \\
a>0, s \in \mathfrak{R} ;
\end{array}
$$

Type II: $\quad \xi_{1}=c+R \cos f(s), \quad \xi_{2}=R \sin f(s)$,

$$
\begin{gathered}
f(s)=2 \tan ^{-1}\left(a e^{b s}\right), \\
c \in \mathfrak{R}, \quad R>0, a>0, b>0, s \in \mathfrak{R} .
\end{gathered}
$$

These are both in affinely parametrized form. In Type II it is simpler to pass to a nonaffine angle type parameter $s$ $\in(0, \pi)$, and replace Eq. (5.24b) with

$$
\text { Type II: } \xi_{1}=c+R \cos s, \quad \xi_{2}=R \sin s,
$$$$
c \in \mathfrak{R}, \quad R>0,0<s<\pi \text {. }
$$

Type I geodesics are straight semi-infinite lines parallel to the $\xi_{2}$ axis. Type II geodesics are semicircles centered on the $\xi_{1}$ axis and lying above this axis.

In each case we can now ask whether a constrained geodesic in $M$ is a null phase curve. As in the previous example of coherent states, here too we emphasize that we are concerned with curves within the manifold of centered normalized Gaussian wave functions, and at no stage with linear combinations of such wave functions. We look at the two types of constrained geodesics in turn and find these results (after simple reparametrizations):

$$
\begin{array}{cl}
\text { Type I: } & \Psi(s)=\psi\left[\xi_{1}=a, \xi_{2}=b s ; \alpha(s)\right], \\
& \arg \left(\Psi(s), \Psi\left(s^{\prime}\right)\right)=0 ;
\end{array}
$$

Type II: $\Psi(s)=\psi\left[\xi_{1}=c+R \cos s, \xi_{2}=R \sin s ; \alpha(s)\right]$,

$$
\arg \left(\Psi(s), \Psi\left(s^{\prime}\right)\right)=\frac{1}{4}\left(s-s^{\prime}\right) .
$$


[In both cases the choice of phase angle $\alpha(s)$ is irrelevant.] So in both cases the criterion (3.4) is obeyed, and both types of curves in $M$ arising from the two types of geodesics in the upper half $\xi$ plane are simultaneously constrained geodesics and null phase curves.

The statement of the generalized connection (3.7) is clear, and for illustration we consider the case of just three vertices. Let $A, A^{\prime}$, and $A^{\prime \prime}$ be any three points in the upper half complex plane, and for any choices of phases $\alpha$ consider the three normalized centered Gaussian states $\psi(A ; \alpha)$, $\psi\left(A^{\prime} ; \alpha^{\prime}\right)$, and $\psi\left(A^{\prime \prime} ; \alpha^{\prime \prime}\right)$. Join $A$ to $A^{\prime}, A^{\prime}$ to $A^{\prime \prime}$, and $A^{\prime \prime}$ to $A$ by a geodesic of Type I or Type II as appropriate in each case. This can always be done, and we obtain a hyperbolic triangle. In $M$ we obtain a triangle with vertices $\rho(A)$ $=\pi[\psi(A ; \alpha)]$ etc., and whose sides are constrained geodesics, and we can state

$$
\begin{gathered}
\varphi_{g}\left[\begin{array}{c}
\text { triangle in } M \text { with vertices } \\
\rho(A), \rho\left(A^{\prime}\right), \rho\left(A^{\prime \prime}\right) \\
\text { and sides as constrained geodesics }
\end{array}\right] \\
=-\arg \Delta_{3}\left[\psi(A ; \alpha), \psi\left(A^{\prime} ; \alpha^{\prime}\right), \psi\left(A^{\prime \prime} ; \alpha^{\prime \prime}\right)\right] .
\end{gathered}
$$

An application of this result has been used elsewhere [17] to show that the classical Gouy phase [18] in wave optics is related to a Bargmann invariant and hence is a geometric phase.

\section{Subset of two-mode coherent states}

In the preceding two examples, we found that while constrained geodesics differed from free geodesics, they were nevertheless null phase curves and so led to important instances of Eq. (3.7). This is, however, fortuitous; the really important objects for our purposes are the null phase curves, and in a given situation constrained geodesics may well not be such curves. In our fourth and final example, dealing with a subset of states for a two-mode system, we will find that this is exactly what happens. However, we will be able to completely determine all null phase curves directly, so that the generalization (3.7) can be meaningfully stated.

For a two-mode system with creation and annihilation operators $\hat{a}_{j}^{+}, \hat{a}_{j}$ obeying the standard commutation relations

$$
\left[\hat{a}_{j}, \hat{a}_{k}^{\dagger}\right]=\delta_{j k}, \quad\left[\hat{a}_{j}, \hat{a}_{k}\right]=\left[\hat{a}_{j}^{\dagger}, a_{k}^{\dagger}\right]=0, j, k=1,2,
$$

the general coherent state is labeled with two independent complex numbers arranged as a column vector $z=\left(z_{1}, z_{2}\right)^{T}$ :

$$
\begin{gathered}
|z\rangle=\exp \left(-\frac{1}{2} z^{\dagger} z+z_{1} \hat{a}_{1}^{\dagger}+z_{2} \hat{a}_{2}^{\dagger}\right)|0\rangle, \\
\hat{a}_{j}|z\rangle=z_{j}|z\rangle, \quad j=1,2 .
\end{gathered}
$$

Within this family of all normalized coherent states, we now define a submanifold (of real dimension three including an overall phase), an " $S^{2}$ worth of states," by taking $\theta, \phi$ to be spherical polar angles on a sphere $S^{2}$ and setting $z_{1}$ and $z_{2}$ equal to the following:

$$
z_{1}=\cos \theta, \quad z_{2}=e^{i \phi} \sin \theta, \quad 0 \leqslant \theta \leqslant \pi, \quad 0 \leqslant \phi \leqslant 2 \pi .
$$

Therefore, the submanifold $\mathcal{M} \subset \mathcal{H}$ is parametrized by $\theta, \phi$ and a phase $\alpha$ and we write

$$
\begin{aligned}
\mathcal{M} & =\{\psi(\theta, \phi ; \alpha) \\
& \left.=e^{i \alpha}\left|\cos \theta, e^{i \phi} \sin \theta\right\rangle \mid 0 \leqslant \theta \leqslant \pi, 0 \leqslant \phi, \alpha \leqslant 2 \pi\right\} \subset \mathcal{H},
\end{aligned}
$$

where the ket on the right is a particular two-mode coherent state with $z^{\dagger} z=1$ :

$$
\psi(\theta, \phi ; \alpha)=\exp \left(i \alpha+\hat{a}_{1}^{\dagger} \cos \theta+\hat{a}_{2}^{\dagger} e^{i \phi} \sin \theta-1 / 2\right)|0\rangle .
$$

Omitting the arguments $\theta, \phi, \alpha$ for simplicity, we easily find

$$
\begin{gathered}
u_{\theta}=\frac{\partial}{\partial \theta} \psi=\left(-\sin \theta \hat{a}_{1}^{\dagger}+e^{i \phi} \cos \theta \hat{a}_{2}^{\dagger}\right) \psi, \\
u_{\phi}=\frac{\partial}{\partial \phi} \psi=i e^{i \phi} \sin \theta \hat{a}_{2}^{\dagger} \psi ; \\
\left(\psi, u_{\theta}\right)=0, \quad\left(\psi, u_{\phi}\right)=i \sin ^{2} \theta ; \\
u_{\theta}^{\perp}=u_{\theta}, \quad u_{\phi}^{\perp}=i \sin \theta\left(e^{i \phi} \hat{a}_{2}^{\dagger}-\sin \theta\right) \psi .
\end{gathered}
$$

Repeatedly exploiting the eigenvector relation (5.29) and its adjoint, we compute the inner products among the vectors in Eq. $(5.33 \mathrm{c})$ :

$$
\left(u_{\theta}^{\perp}, u_{\theta}^{\perp}\right)=1, \quad\left(u_{\theta}^{\perp}, u_{\phi}^{\perp}\right)=i \cos \theta \sin \theta, \quad\left(u_{\phi}^{\perp}, u_{\phi}^{\perp}\right)=\sin ^{2} \theta .
$$

Taking the real parts here, we see that the metric induced on $M=\pi[\mathcal{M}] \sim S^{2}$ in $\mathcal{R}$, parametrized by angles $\theta$ and $\phi$, is just the usual rotationally invariant one:

$$
g_{\theta \theta}(\theta, \phi)=1, g_{\theta \phi}=0, g_{\phi \phi}(\theta, \phi)=\sin ^{2} \theta .
$$

The corresponding constrained geodesics are therefore simply great-circle arcs. The question is whether they lead to null phase curves in $M$ and $\mathcal{M}$.

A general parametrized great-circle arc on $S^{2}$ is traced out by an $s$-dependent unit vector $\hat{n}(s)$ with polar angles $\theta(s), \phi(s)$ :

$$
\begin{aligned}
\hat{n}(s) & =\hat{a} \cos s+\hat{b} \sin s \\
& =[\sin \theta(s) \cos \phi(s), \quad \sin \theta(s) \sin \phi(s), \cos \theta(s)], \\
& \hat{a}, \hat{b} \in S^{2}, \hat{a} \cdot \hat{b}=0 .
\end{aligned}
$$

The corresponding constrained geodesic $\mathcal{C}_{\text {constr. geo. }} \subset \mathcal{M}$ (omitting the phase $\alpha$ ) is the curve of coherent states 


$$
\begin{gathered}
\Psi(s)=\left|z_{1}(s), z_{2}(s)\right\rangle, \\
z_{1}(s)=\cos \theta(s)=a_{3} \cos s+b_{3} \sin s, \\
z_{2}(s)=e^{i \phi(s)} \sin \theta(s)=\left(a_{1}+i a_{2}\right) \cos s+\left(b_{1}+i b_{2}\right) \sin s .
\end{gathered}
$$

To see if this is a null phase curve we compute the phase of $\left(\Psi(s), \Psi\left(s^{\prime}\right)\right)$ :

$$
\begin{aligned}
\arg \left(\Psi(s), \Psi\left(s^{\prime}\right)\right)= & \arg \left\langle z_{1}(s), z_{2}(s) \mid z_{1}\left(s^{\prime}\right), z_{2}\left(s^{\prime}\right)\right\rangle \\
= & \arg \left\{\exp \left(z_{1}(s) z_{1}\left(s^{\prime}\right)+z_{2}(s) * z_{2}\left(s^{\prime}\right)\right)\right\} \\
= & \arg \left\{\operatorname { e x p } \left(\left[\left(a_{1}-i a_{2}\right) \cos s\right.\right.\right. \\
& \left.+\left(b_{1}-i b_{2}\right) \sin s\right]\left[\left(a_{1}+i a_{2}\right) \cos s^{\prime}\right. \\
& \left.\left.\left.+\left(b_{1}+i b_{2}\right) \sin s^{\prime}\right]\right)\right\} \\
= & (\hat{a} \wedge \hat{b})_{3} \sin \left(s^{\prime}-s\right) .
\end{aligned}
$$

Unless it vanishes, this is not a separable function of $s^{\prime}$ and $s$. We conclude that the geodesic (5.36) on $S^{2}$ leads to a constrained geodesic $\mathcal{C}_{\text {constr.geo. }} \subset \mathcal{M}$, which is, in general, not a null phase curve. The only exception is when $(\hat{a} \wedge \hat{b})_{3}=0$, that is, the geodesic (5.36) on $S^{2}$ lies on a meridian of longitude, with $\hat{a} \wedge \hat{b}$ being a vector in the 1-2 plane.

On the other hand, in this example it is quite easy to explicitly find all null phase curves on $M($ and $\mathcal{M})$ ! Let $\Gamma$ $=\{\hat{n}(s)\} \subset S^{2}$ be given, and let us consider the induced curve $\mathcal{C}_{\Gamma}$ in $\mathcal{M}$ :

$$
\begin{aligned}
\mathcal{C}_{\Gamma} & =\left\{\Psi_{\Gamma}(s)=\left|n_{3}(s), n_{1}(s)+i n_{2}(s)\right\rangle\right. \\
& \left.=\exp \left(-\frac{1}{2}+n_{3}(s) \hat{a}_{1}^{\dagger}+\left(n_{1}(s)+i n_{2}(s)\right) \hat{a}_{2}^{\dagger}\right)|0\rangle\right\} .
\end{aligned}
$$

We find that

$$
\arg \left(\Psi_{\Gamma}(s), \Psi_{\Gamma}\left(s^{\prime}\right)\right)=\left[\hat{n}(s) \wedge \hat{n}\left(s^{\prime}\right)\right]_{3}
$$

This will be a separable function of $s^{\prime}$ and $s$ if and only if, for some constants $\beta$ and $\gamma$, we have

$$
n_{2}(s)=\beta n_{1}(s)+\gamma
$$

The geometrical interpretation of this is that the projection of $\Gamma$ on the 1-2 plane must be a straight line. In that case, $\mathcal{C}_{\Gamma}$ is indeed a null phase curve in $\mathcal{M}$, as we have

$$
\arg \left(\Psi_{\Gamma}(s), \Psi_{\Gamma}\left(s^{\prime}\right)\right)=\gamma\left[n_{1}(s)-n_{1}\left(s^{\prime}\right)\right],
$$

which is separable in $s^{\prime}$ and $s$. One can easily see that each such $\Gamma$ is a latitude circle arc on $S^{2}$ corresponding to (i.e., perpendicular to) some axis lying in the 1-2 plane, and given any two points on $S^{2}$, we can always connect them by such a $\Gamma$. In other words, such $\Gamma$ are intersections of $S^{2}$ with planes perpendicular to the 1-2 plane. When such a latitude circle arc is also a great-circle arc, we recover the result of the previous paragraph.

The upshot of this example is that here we have a nontrivial illustration of the difference between constrained geodesics and null phase curves. However, since we have been able to find all of the latter, and any two points in $\mathcal{M}$ can be connected by some null phase curve, we have succeeded in providing a nontrivial two-mode example of the generalized connection (3.7), without using constrained geodesics.

\section{RAY SPACE AND DIFFERENTIAL GEOMETRIC FORMULATIONS}

Very soon after the discovery of the geometric phase, the differential geometric expressions of its structure and significance were developed $[19,3-5,11]$, by relating it to anholonomy and curvature in a suitable Hermitian line bundle in quantum mechanical ray space. In this section we provide a brief discussion of the properties and uses of the new concept of null phase curves at the ray space level and also in the differential geometric language. Only necessary background material will be recalled, and derivations will be omitted. Since they may be useful for practical calculations, where possible local coordinate expressions of important differential geometric objects will be given.

From the preceding sections it is evident that for our purposes it is important to deal with open null phase curves in general, since it is through them that the connection (3.7) of the Bargmann invariants to geometric phases is made. Their definition (3.4) in terms of Hilbert space lifts is quite simple. Nevertheless, it is of interest to develop a direct ray space formulation; this can be done essentially via the Bargmann invariants themselves. From their definition (2.8), it is clear that any $\triangle_{2}$ is real nonnegative, while $\triangle_{n}$ 's for $n \geqslant 3$ are, in general, complex. On the other hand, it is also known that any $\triangle_{n}$ for $n \geqslant 4$ can be written as the ratio of a suitable product of $\triangle_{3}$ 's and a suitable product of $\triangle_{2}$ 's:

$$
\begin{aligned}
\triangle_{n}\left(\psi_{1}, \psi_{2}, \cdots, \psi_{n}\right) \\
\quad=\prod_{j=3}^{n} \triangle_{3}\left(\psi_{1}, \psi_{j-1}, \psi_{j}\right) / \prod_{j=4}^{n} \triangle_{2}\left(\psi_{1}, \psi_{j-1}\right) .
\end{aligned}
$$

In this sense the three-vertex Bargmann invariant $\triangle_{3}$ is the basic or primitive one as far as phases are concerned. [The basic cyclic invariance of $\triangle_{n}\left(\psi_{1}, \psi_{2}, \cdots, \psi_{n}\right)$ is not manifest in Eq. (6.1), but it is not lost either.] Guided by these facts, we give now a direct ray space characterization of null phase curves.

If $C=\{\rho(s)\} \subset \mathcal{R}$ is a null phase curve and $\mathcal{C}^{(h)}$ $=\left\{\psi^{(h)}(s)\right\}$ is a horizontal Hilbert space lift obeying Eq. (3.4), we see immediately that for any choices of parameter values $s, s^{\prime}, s^{\prime \prime}$,

$$
\begin{aligned}
& \triangle_{3}\left[\psi^{(h)}(s), \psi^{(h)}\left(s^{\prime}\right), \psi^{(h)}\left(s^{\prime \prime}\right)\right]=\operatorname{Tr}\left\{\rho(s) \rho\left(s^{\prime}\right) \rho\left(s^{\prime \prime}\right)\right\} \\
& \quad=\text { real and } \geqslant 0
\end{aligned}
$$

and so also for any $n$ parameter values $s_{1}, s_{2}, \ldots, s_{n}$, from Eq. (6.1),

$$
\begin{aligned}
\triangle_{n}\left[\psi^{(h)}\left(s_{1}\right), \ldots, \psi^{(h)}\left(s_{n}\right)\right] & =\operatorname{Tr}\left\{\rho\left(s_{1}\right) \ldots \rho\left(s_{n}\right)\right\} \\
& =\text { real and } \geqslant 0 .
\end{aligned}
$$

As a consequence, by differentiation with respect to $s_{2}, \ldots, s_{n}$ we have 


$$
\operatorname{Tr}\left\{\rho\left(s_{1}\right) \frac{d \rho\left(s_{2}\right)}{d s_{2}} \ldots \frac{d \rho\left(s_{n}\right)}{d s_{n}}\right\}=\text { real. }
$$

Now, it is known that the geometric phase for any connected portion of any $C$ can be expressed directly in terms of $\rho(s)$ as follows, whether or not $C$ is a null phase curve:

$$
\begin{aligned}
\varphi_{g}\left[\rho\left(s_{1}\right) \text { to } \rho\left(s_{2}\right) \text { along } C\right] \\
=\arg \left[\operatorname{Tr}\left\{\rho\left(s_{1}\right) P\left(\exp \int_{s_{1}}^{s_{2}} d s \frac{d \rho(s)}{d s}\right)\right\}\right] \\
=\arg \left[1+\sum_{n=1}^{\infty} \int_{s_{1}}^{s_{2}} d s_{n}^{\prime} \int_{s_{1}}^{s_{n}^{\prime}} d s_{n-1}^{\prime} \cdots \int_{s_{1}}^{s_{2}^{\prime}} d s_{1}^{\prime}\right. \\
\left.\quad \times \operatorname{Tr}\left\{\rho\left(s_{1}\right) \frac{d \rho\left(s_{n}^{\prime}\right)}{d s_{n}^{\prime}} \ldots \frac{d \rho\left(s_{1}^{\prime}\right)}{d s_{1}^{\prime}}\right\}\right],
\end{aligned}
$$

where $P$ is the ordering symbol placing later parameter values to the left of earlier ones. If Eq. (6.2) holds on $C$ [and so as a consequence Eqs. (6.3) and (6.4) as well], we see that at every stage only real quantities are involved, the geometric phase in Eq. (6.5) vanishes, and $C$ is a null phase curve. This leads to the ray space characterization of null phase curves we are seeking:

$$
\begin{aligned}
C= & \{\rho(s)\} \subset \mathcal{R} \text { is a null phase curve } \\
& \Leftrightarrow \operatorname{Tr}\left\{\rho(s) \rho\left(s^{\prime}\right) \rho\left(s^{\prime \prime}\right)\right\} \\
= & \text { real nonnegative, any } s, s^{\prime}, s^{\prime \prime} .
\end{aligned}
$$

Turning now to the specific differential geometric aspects, it is well known that while the dynamical phase $\varphi_{\text {dyn }}[\mathcal{C}]$ is an additive quantity, $\varphi_{g}[C]$ does not have this property. On the manifold of unit vectors in Hilbert space $\mathcal{H}$, there is a oneform $A$ such that

$$
\varphi_{\mathrm{dyn}}[\mathcal{C}]=\int_{\mathcal{C}} A
$$

However, referring to the projection $\pi: \mathcal{H} \rightarrow \mathcal{R}, A$ is not the pull-back via $\pi^{*}$ of any one-form on the space of unit rays, and $\varphi_{g}[C]$ is not the integral along $C$ of any one-form on $\mathcal{R}$. In fact, this lack of additivity can be expressed via the Bargmann invariant $\triangle_{3}$. If $C_{12}$ connects $\rho_{1}$ to $\rho_{2}$ in $\mathcal{R}$ and $C_{23}$ connects $\rho_{2}$ to $\rho_{3}$, then $C_{12} \cup C_{23}$ runs from $\rho_{1}$ to $\rho_{3}$ and

$$
\begin{gathered}
\varphi_{g}\left[C_{12} \cup C_{23}\right]=\varphi_{g}\left[C_{12}\right]+\varphi_{g}\left[C_{23}\right]-B_{3}\left(\psi_{1}, \psi_{2}, \psi_{3}\right), \\
B_{3}\left(\psi_{1}, \psi_{2}, \psi_{3}\right)=\arg \triangle_{3}\left(\psi_{1}, \psi_{2}, \psi_{3}\right) .
\end{gathered}
$$

More generally, for an (generally) open curve consisting of $(n-1)$ pieces $C_{12}, C_{23}, \cdots C_{n-1, n}$ joining $\rho_{1}$ to $\rho_{2}, \rho_{2}$ to $\rho_{3}, \cdots, \rho_{n-1}$ to $\rho_{n}$, we generalize Eq. (6.8) to the following:

$$
\begin{aligned}
\varphi_{g}\left[C_{12} \cup C_{23} \cup \cdots \cup C_{n-1, n}\right]= & \sum_{j=1}^{n-1} \varphi_{g}\left[C_{j, j+1}\right] \\
& -B_{n}\left(\psi_{1}, \psi_{2}, \cdots, \psi_{n}\right),
\end{aligned}
$$

$$
\begin{aligned}
B_{n}\left(\psi_{1}, \psi_{2}, \cdots, \psi_{n}\right) & =\arg \triangle_{n}\left(\psi_{1}, \psi_{2}, \ldots, \psi_{n}\right) \\
& =\sum_{j=3}^{n} B_{3}\left(\psi_{1}, \psi_{j-1}, \psi_{j}\right) .
\end{aligned}
$$

If we connect $\rho_{n}$ back to $\rho_{1}$ via $C_{n, 1}$ to get a closed curve of $n$ pieces, then we have the specific result

$$
\begin{aligned}
\varphi_{g} & {\left[C_{12} \cup C_{23} \cup \cdots \cup C_{n-1, n}, \cup C_{n, 1}\right] } \\
= & \varphi_{g}\left[C_{12}\right]+\varphi_{g}\left[C_{23}\right]+\cdots+\varphi_{g}\left[C_{n, 1}\right] \\
& -B_{n}\left(\psi_{1}, \psi_{2}, \cdots, \psi_{n}\right) .
\end{aligned}
$$

Compared to Eq. (6.9), we have one extra $\varphi_{g}$ term on the right-hand side, but the Bargmann phase term $B_{n}$ is the same. We see that the lack of additivity shown in all Eqs. $(6.8,6.9,6.10)$ is due to the Bargmann pieces. There is, however, an exception to this general nonadditivity, which occurs in Eq. (6.8) when $\rho_{3}=\rho_{1}$ and $C_{12} \cup C_{23}$ is a closed loop. Then we find

$$
\begin{gathered}
\partial\left(C_{12} \cup C_{23}\right)=0, \rho_{3}=\rho_{1}: \\
\varphi_{g}\left[C_{12} \cup C_{21}\right]=\varphi_{g}\left[C_{12}\right]+\varphi_{g}\left[C_{21}\right], \\
\text { i.e., } \varphi_{g}\left[C_{12}\right]=\varphi_{g}\left[C_{12} \cup C_{21}\right]-\varphi_{g}\left[C_{23}\right] .
\end{gathered}
$$

In the past, this result has been used [4] to relate $\varphi_{g}[C]$ for an open $C$ to $\varphi_{g}\left[C \cup C^{\prime}\right]$ for a closed $C \cup C^{\prime}$ by choosing $C^{\prime}$ to be a free geodesic, for then $\varphi_{g}\left[C^{\prime}\right]=0$. Now we can generalize this process: if $C$ is an open curve from $\rho_{1}$ to $\rho_{2}$ in $\mathcal{R}$, and $C^{\prime}$ is any null phase curve from $\rho_{2}$ back to $\rho_{1}$, we have the result

$$
\varphi_{g}[\text { open curve } C]=\varphi_{g}\left[\text { closed loop } C \cup C^{\prime}\right] .
$$

This is the most general way in which an open curve geometric phase can be reduced to a closed loop geometric phase. More generally, comparing Eqs. $(6.9,6.10)$ valid for generally open and for a closed curve, we see that if the last piece $C_{n, 1}$ is a null phase curve, we convert an open curve geometric phase to a closed loop geometric phase:

$$
\begin{aligned}
& \varphi_{g}\left[C_{12} \cup C_{23} \cup \cdots \cup C_{n-1, n}\right] \\
& \quad=\varphi_{g}\left[C_{12} \cup C_{23} \cup \cdots \cup C_{n-1, n} \cup C_{n, 1}\right] .
\end{aligned}
$$

At this point it is natural to express a closed loop geometric phase as a suitable "area integral" of a two-form, both at Hilbert and ray space levels. Whereas $A$ is not the pull-back of any one-form on $\mathcal{R}$, we do have $d A=\pi^{*} \omega$, where $\omega$ is a symplectic (closed, nondegenerate) two-form on $\mathcal{R}$. Then, if $\mathcal{C}$ is a closed loop in $\mathcal{H}, \partial \mathcal{C}=0$, so that $C=\pi(\mathcal{C})$ is a closed loop in $\mathcal{R}$, we have

$$
\varphi_{g}[C]=\int_{\mathcal{S}} d A=\int_{S} \omega,
$$

where $\mathcal{S}$ and $S=\pi(\mathcal{S})$ are two-dimensional surfaces in $\mathcal{H}$ and $\mathcal{R}$, respectively, with boundaries $\mathcal{C}$ and $C$ :

$$
\partial \mathcal{S}=\mathcal{C}, \quad \partial S=C .
$$


With the help of local coordinates on $\mathcal{H}$ and $\mathcal{R}$ we get explicit expressions for $A, d A$ and $\omega$. Around any point $\rho_{0}$ $\in \mathcal{R}$, and for some chosen $\psi_{0} \in \pi^{-1}\left(\rho_{0}\right)$, we define an (open) neighborhood $N \subset \mathcal{R}$ by

$$
N=\left\{\rho \in \mathcal{R} \mid \operatorname{Tr}\left(\rho_{0} \rho\right)>0\right\} .
$$

We can introduce real independent coordinates over $N$ as follows. Let $\left\{\psi_{0}, e_{1}, e_{2}, \ldots, e_{r}, \ldots\right\}$ be an orthonormal basis for $\mathcal{H}$. Then points in $N$ can be "labeled"' in a one-to-one manner with vectors $\mathcal{X} \in \mathcal{H}$ orthogonal to $\psi_{0}$ and with norm less than unity:

$$
\begin{gathered}
\chi(\beta, \gamma)=\frac{1}{\sqrt{2}} \sum_{r}\left(\beta_{r}-i \gamma_{r}\right) e_{r}, \\
\|\chi(\beta, \gamma)\|^{2}=\frac{1}{2} \sum_{r}\left(\beta_{r}^{2}+\gamma_{r}^{2}\right)<1: \\
\psi(\beta, \gamma)=\chi(\beta, \gamma)+\sqrt{1-\|\chi(\beta, \gamma)\|^{2}} \psi_{0}, \\
\rho \in N \Leftrightarrow \rho=\psi(\beta, \gamma) \psi(\beta, \gamma)^{\dagger}, \text { for some } \beta, \gamma .
\end{gathered}
$$

Thus the real independent $\beta$ 's and $\gamma$ 's, subject to the inequality above, are local coordinates for $N$. They can be extended to get local coordinates for $\pi^{-1}(N) \subset \mathcal{H}$ by including a phase angle $\alpha$ :

$$
\psi \in \pi^{-1}(N) \Leftrightarrow \psi=\psi(\alpha ; \beta, \gamma)=e^{i \alpha} \psi(\beta, \gamma), \quad 0 \leqslant \alpha<2 \pi .
$$

In these local coordinates over $N$ and $\pi^{-1}(N)$ we have the expressions

$$
\begin{gathered}
A=d \alpha+\frac{1}{2} \sum_{r}\left(\gamma_{r} d \beta_{r}-\beta_{r} d \gamma_{r}\right), \\
d A=\sum_{r} d \gamma_{r} \wedge d \beta_{r}, \\
\omega=\sum_{r} d \gamma_{r} \wedge d \beta_{r} .
\end{gathered}
$$

The closure and nondegeneracy of $\omega$ are manifest, so it is a symplectic two-form on $\mathcal{R}$; and the coordinates $\beta, \gamma$ realize the local Darboux or canonical structure for it. On the other hand, in these "symplectic" coordinates the Fubini-Study metric is a bit involved. If we combine the $\beta$ 's and $\gamma$ 's into a single column vector $\eta=\left(\beta_{1} \beta_{2} \ldots \gamma_{1} \gamma_{2} \ldots\right)^{T}$, then the length functional $L[C]$ of Eq. (2.5) assumes the following local form:

$$
\begin{aligned}
& L[C]=\int d s \sqrt{\dot{\eta}^{T} g(\eta) \dot{\eta}},
\end{aligned}
$$

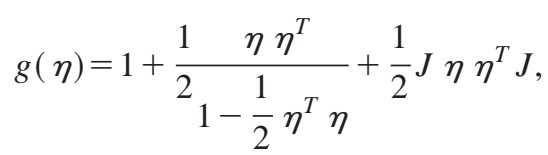

$$
\begin{aligned}
& J=\left(\begin{array}{cc}
0 & 1 \\
-1 & 0
\end{array}\right), \quad \eta^{T} \eta<2 .
\end{aligned}
$$

The symplectic matrix $J$ plays a role in this expression for the metric tensor matrix $g(\eta)$. This matrix $g(\eta)$ is verified to be real symmetric positive definite, since one eigenvalue is $\left(1-\frac{1}{2} \eta^{T} \eta\right)^{-1}$ (eigenvector $\eta$ ), another eigenvalue is (1 $-\frac{1}{2} \eta^{T} \eta$ ) (eigenvector $J \eta$ ), and the remaining eigenvalues are all unity. We appreciate that for considerations of geometric phases and null phase curves this kind of local description is really appropriate, while free geodesics appear unavoidably complicated.

We also notice that, when $\mathcal{H}$ is finite dimensional and the real dimension of the space $\mathcal{R}$ of unit rays is $2 n$, the symplectic two-form $\omega$ of Eq. (6.19) is invariant under the linear matrix group $S p(2 n, R)$ acting on the local coordinates $\beta, \gamma$. On the other hand, the integrand of the length functional $L[C]$ in Eq. (6.20) possesses invariance only under $\operatorname{Sp}(2 n, R) \cap S O(2 n) \simeq U(n)$, which is just the group of changes in the choice of the vectors $\left\{e_{r}\right\}$ which together with $\psi_{0}$ make up an orthonormal basis for $\mathcal{H}$.

Returning now to the discussions in Secs. III and IV, we can bring in submanifolds $M \subset \mathcal{R}, \mathcal{M}=\pi^{-1}(M) \subset \mathcal{H}$, with local coordinates $\xi^{\mu}, \alpha$ as indicated in Eqs. (4.1) and (4.2). Let $i_{\mathcal{M}}: \mathcal{M} \subset \mathcal{H}$ and $i_{M}: M \subset \mathcal{R}$ be the corresponding identification maps. Straightforward calculations show that the pull-backs of $A, d A, \omega$ in Eq. (6.15) to $\mathcal{M}$ and $M$ are locally given (with mild abuse of notation) by

$$
\begin{gathered}
i_{\mathcal{M}}^{*} A=d \alpha+\operatorname{Im}\left(\psi(\xi ; \alpha), u_{\mu}(\xi ; \alpha)\right) d \xi^{\mu} \\
i_{\mathcal{M}}^{*} d A=i_{M}^{*} \omega=\operatorname{Im}\left(u_{\mu}^{\perp}(\xi ; \alpha), u_{\nu}^{\perp}(\xi ; \alpha)\right) d \xi^{\mu} \wedge d \xi^{\nu} \\
=\operatorname{Im}\left[u_{\mu}(\xi ; \alpha), u_{\nu}(\xi ; \alpha)\right] d \xi^{\mu} \wedge d \xi^{\nu}
\end{gathered}
$$

We see, as is well known, that while the real symmetric part of the Hermitian matrix $\left[\left(u_{\mu}^{\perp}, u_{\nu}^{\perp}\right)\right]$ determines the metric, Eq. (4.7), the imaginary antisymmetric part of the same matrix is relevant for symplectic structure and geometric phase, reinforcing the link between the latter two. [When $M=\mathcal{R}$ and $\mathcal{M}=\mathcal{H}$, the $\xi^{\mu}$ 's become the $\beta$ 's and $\gamma$ 's of Eqs. (6.17), and we immediately recover the expressions (6.19).] For our present purposes, the following comments are pertinent. While $\omega$ is closed and nondegenerate, $i_{M}^{*} \omega$ is closed but may well be degenerate. An extreme case is when $M$ is an isotropic submanifold in $\mathcal{R}$, for then $i_{M}^{*} \omega=0$. Such a situation can easily arise if, for example, $M$ is described by a family of real Schrödinger wave functions $\psi(\xi ; q)$. (A Lagrangian submanifold in $\mathcal{R}$ is a particular case of an isotropic submanifold when the dimension is maximal, namely, half the real dimension of $\mathcal{R}$.) One may expect that if $M$ is isotropic and $C \subset M$, then $C$ is a null phase curve. However, this need not always be so, and the situation is as follows. For a general open curve $C_{12}$ from $\rho_{1}$ to $\rho_{2}$ in a general submanifold $M$, if we can find a null phase curve $C_{21}$ from $\rho_{2}$ to $\rho_{1}$ also lying in $M$, then $C_{12} \cup C_{21}$ is a closed loop; if $\pi_{1}(M)$ $=0$, we can find a two-dimensional surface $S \in M$ having $C_{12} \cup C_{21}$ as boundary. Then from Eq. (6.12) we obtain under these circumstances

$$
\varphi_{g}\left[C_{12}\right]=\varphi_{g}\left[C_{12} \cup C_{21}\right]=\int_{S \in M} i_{M}^{*} \omega
$$


Here, as stated above, we had to choose $C_{21}$ to be a null phase curve. (When $\rho_{2}=\rho_{1}$ and $C_{12}$ is already a closed loop, there is no need for any $C_{21}$; it can be chosen to be trivial!) If, however, $M$ is an isotropic submanifold, i.e., $i_{M}^{*} \omega=0$ [and assuming also $\pi_{1}(M)=0$ ], we can extract some very interesting consequences for geometric phases, though it falls short of the vanishing of $\varphi_{g}[C]$ for every $C \subset M$. We have the chain of implications

$$
\begin{aligned}
i_{M}^{*} \omega=0 & \Leftrightarrow \int_{S} i_{M}^{*} \omega=0, \text { any two dimensional } S \subset M \\
& \Leftrightarrow \varphi_{g}\left[C_{12} \cup C_{21}\right]=0, \text { any } \rho_{1}, \rho_{2}, C_{12}, C_{21} \text { in } M \\
& \Leftrightarrow \varphi_{g}\left[C_{12}\right] \text { unchanged under any continuous deformation of } C_{12} \text { leaving the end points } \rho_{1}, \rho_{2} \text { fixed }
\end{aligned}
$$

Thus, within an isotropic submanifold, the geometric phase for a general curve depends on the two end points alone. When the curve chosen is closed, it can be continuously shrunk to a point [since, $\pi_{1}(M)=0$ ] and then its geometric phase vanishes. One can thus say in summary:

$$
\begin{gathered}
M \subset \mathcal{R}, i_{M}^{*} \omega=0, \pi_{1}(M)=0, C \subset M: \\
\varphi_{g}[C]=0 \text { if } \partial C=0 ; \\
\varphi_{g}[C]=\text { function of } \partial C \text { alone, if } \partial C \neq 0 .
\end{gathered}
$$

The main conclusion is that general open curves in an isotropic submanifold need not be null phase curves, but geometric phases are invariant under continuous changes of their arguments, leaving the end points unchanged. Perhaps this is not too surprising after all, since the isotropic property is a two-form condition.

\section{CONCLUDING REMARKS}

We have shown that the familiar connection between the Bargmann invariants and geometric phases in quantum mechanics, based on the properties of free geodesics in ray and Hilbert spaces, can be generalized to a very significant extent. In fact we have shown that our generalization is the broadest one possible. The essential concept is that of null phase curves in Hilbert and ray spaces-the replacement of free geodesics by such curves leads to our generalization. We have seen through examples that this wider connection between Bargmann invariants and geometric phases is just what is needed in several physically relevant situations.

Motivated by the fact that free geodesics are always null phase curves, we have defined the concept of constrained geodesics and posed the problem of determining when these may be null phase curves. We have presented two examples when this is indeed so and one where they are not the same. This re-emphasizes the fact that constrained geodesics and null phase curves are, in principle, different objects, and intensifies the need to find useful characterizations of the former which may ensure the latter property for them. This is certain to shed more light on the general questions raised in this paper, and we plan to return to them in the future.

\section{ACKNOWLEDGMENTS}

One of us (E.M.R.) thanks the Third World Academy of Sciences, Trieste, Italy for their financial support, the JNCASR, Bangalore, India for support, and the Center for Theoretical Studies, IISc., Bangalore for providing facilities during the completion of this work.
[1] M. V. Berry, Proc. R. Soc. London, Ser. A 392, 45 (1984).

[2] Many of the early papers on geometric phase have been reprinted in Geometric Phases in Physics, edited by A. Shapere and F. Wilczek (World Scientific, Singapore, 1989), and in Fundamentals of Quantum Optics, edited by G. S. Agarwal, SPIE Milestone Series (SPIE, Bellington, 1995).

[3] Y. Aharonov and J. Anandan, Phys. Rev. Lett. 58, 1593 (1987).

[4] J. Samuel and R. Bhandari, Phys. Rev. Lett. 60, 2339 (1988).

[5] F. Wilczek and A. Zee, Phys. Rev. Lett. 52, 2111 (1984).

[6] N. Mukunda and R. Simon, Ann. Phys. (N.Y.) 228, 205 (1993); , 228, 269 (1993).

[7] W. R. Hamilton (unpublished); L. C. Biedenharn and J. D. Louck, Angular Momentum in Quantum Physics. Encyclopedia of Mathematics and its applications (Addison-Wesley, Read- ing, MA, 1981), Vol. 8. Hamilton's theory of turns has been generalized to the simplest noncompact semisimple group $S U(1,1) \sim S L(2, R)$ in R. Simon, N. Mukunda, and E. C. G. Sudarshan, Phys. Rev. Lett. 62, 1331 (1989); J. Math. Phys. 30, 1000 (1989); S. Chaturvedi, V. Srinivasan, R. Simon, and N. Mukunda (unpublished).

[8] R. Simon and N. Mukunda, J. Phys. A: Math. Gen. 25, 6135 (1992).

[9] V. Bargmann, J. Math. Phys. 5, 862 (1964).

[10] E. P. Wigner, Group Theory (Academic, NY, 1959); J. Samuel, Pramana, J. Phys. 48, 959 (1997).

[11] D. Page, Phys. Rev. A 36, 3479 (1987).

[12] S. Kobayashi and K. Nomizu, Foundations of Differential Geometry (Interscience, NY, 1969), Vol. II, Chap. IX.

[13] S. Pancharatnam, Proc.-Indian Acad. Sci., Sect. A 44, 247 
(1956); See also, S. Ramaseshan and R. Nityananda, Curr. Sci. 55, 1225 (1986); M. V. Berry, J. Mod. Opt. 34, 1401 (1987).

[14] G. Khanna, S. Mukhopadhyay, R. Simon, and N. Mukunda, Ann. Phys. (N.Y.) 253, 55 (1997); Arvind, K. S. Mallesh, and N. Mukunda, J. Phys. A: Math. Gen. 30, 2417 (1997).

[15] S. Chaturvedi, M. S. Sriram, and V. Srinivasan, J. Phys. A 20, L1071 (1987).

[16] M. Berger, Geometry II (Springer-Verlag, Berlin, 1987), Chap.
19; G. A. Jones and D. Singerman, Complex Functions: An Algebraic and Geometric Viewpoint (Cambridge University Press, Cambridge, 1987), Chap. 5.

[17] R. Simon and N. Mukunda, Phys. Rev. Lett. 70, 880 (1993).

[18] G. Gouy, C. R. Hebd. Seances Acad. Sci. 110, 125 (1890); A. E. Siegman, Lasers (Oxford University Press, Oxford, 1986), Chap. 17.

[19] B. Simon, Phys. Rev. Lett. 51, 2167 (1983). 